\title{
Alkylative coupling of enaminones and benzimidazole
}

\author{
Milena Staykova, Stela Statkova-Abeghe,* Plamen Angelov, and Iliyan Ivanov \\ Department of Organic Chemistry, University of Plovdiv, 24 “Tzar Asen” Str., 4000, \\ Plovdiv, Bulgaria \\ E-mail: stab@uni-plovdiv.bg
}

\begin{abstract}
$\mathrm{N}$-Acyliminium reagents derived from benzimidazole have been used successfully in C-C bond forming reactions with $\beta$-enaminones. The new 2 -substituted derivatives of 2,3dihydrobenzimidazole are interesting both from synthetic point of view and as potential bioactive compounds.
\end{abstract}

Keywords: $N$-Acyliminium, $\alpha$-amidoalkylation, enaminones

\section{Introduction}

The $\alpha$-amidoalkylation of carbon nucleophiles with $N$-acyliminium compounds is a longestablished method for $C-C$ bond formation. ${ }^{1-4}$ This reaction can be used for the synthesis of various heterocyclic derivatives.

Benzimidazole ring is an important heterocyclic pharmacophore in drug discovery. Benzimidazoles are regarded as a promising class of bioactive heterocyclic compounds that exhibit a range of biological activities - antiviral, ${ }^{5,6}$ antitumor, ${ }^{7,8}$ anticancer, ${ }^{9}$ antimicrobial activity against Staphylococcus aureus, Bacillus subtilis, Escherichia coli and Candida albicans, ${ }^{10}$ antibacterial activity against Staphylococcus aureus, Escherichia coli, Enterococcus faecalis and fungicidal activity against Candida albicans and Asperigillus. ${ }^{11-13}$ Benzimidazoles are potential enterovirus inhibitors. ${ }^{14}$

We have previously used adducts of cyclic imines and acyl chlorides as electrophilic reagents in an intermolecular $\alpha$-amidoalkylation reaction toward aromatics and methylene active carbonyl compounds. ${ }^{15-18}$ Now the same strategy has been successfully applied for coupling of benzimidazole and cyclic enaminones. 


\section{Results and Discussion}

Enaminones, as defined by Greenhill, are monoenamines of 1,3-dicarbonyl compounds ${ }^{19}$ which combine the ambident electrophilicity of enones and the ambident nucleophilicity of enamines. The enaminones 4 necessary for this study were prepared by condensation of dimedone and various primary amines. The reactions were carried out in dichloroethane (or toluene for $\mathbf{4 e}$ ) at reflux temperature and the water was removed with Dean-Stark trap. With low-boiling amines such as methyl- and ethylamine the reaction was carried out at room temperature for $24 \mathrm{~h}$ in the presence of $\mathrm{Na}_{2} \mathrm{SO}_{4}$.

The $\mathrm{N}$-acyliminium reagents $\mathbf{3}$ were obtained after initial $\mathrm{N}$-acylation of benzimidazole $\mathbf{1}$ with alkyl chloroformates $2(\mathrm{R} \mathrm{Me}, \mathrm{Et})$ in the presence of $\mathrm{Et}_{3} \mathrm{~N}$, acting as hydrogen chloride acceptor (Scheme 1). The initial acylation took place at $0{ }^{\circ} \mathrm{C}$ for 15 minutes. Then the reaction mixture was brought to room temperature and second equivalent of alkyl chloroformate was added. In this manner we got the acyliminium reagents $\mathbf{3}$ (Scheme 1).

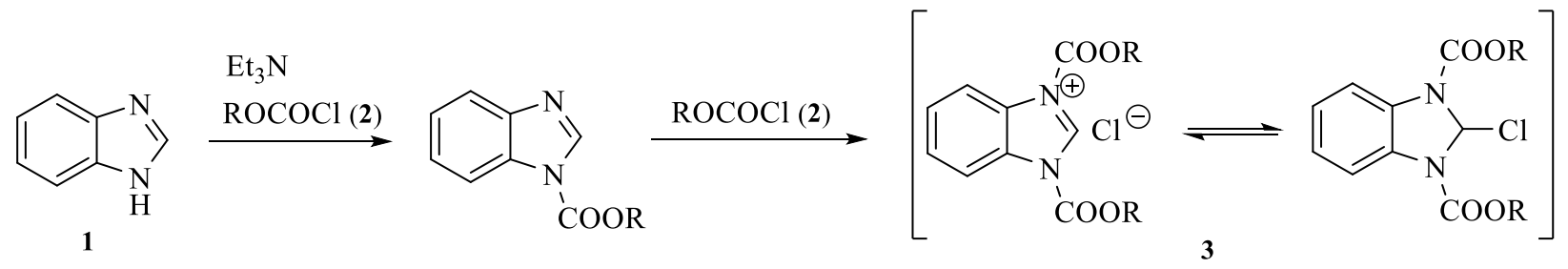

Scheme 1. Preparation of the acyliminium reagents from benzimidazole and alkylchloroformates.

The reactions of the acyliminium reagents 3 with enaminones were carried out in the same reaction vessel for $1 \mathrm{~h}$ at $\mathrm{r}$.t. and in all cases led to a single product $(\mathbf{5} \mathbf{a}-\mathbf{0})$. The substituent $\mathrm{R}_{1}$ was varied in the series of enaminones as indicated in Table 1. Attempts to amidoalkylate acyclic enaminones were not successful even at elevated temperature. The yields with cyclic enaminones 4 were from $11 \%\left(\mathrm{R}^{1}=\mathrm{CH}_{3}\right)$ to $58 \%\left(\mathrm{R}^{1}=\mathrm{C}_{6} \mathrm{H}_{5} \mathrm{CH}_{2}\right)$ and all of the studied examples proceeded regioselectively at the $\alpha$-carbon of the enaminone as indicated by the disappearance of the characteristic vinyl signal in the ${ }^{1} \mathrm{H}-\mathrm{NMR}$ spectra. No reaction at $N$ or $O$ was detected. The main problem limiting the yields was the formation of $\mathrm{HCl}$ salts of the enaminones in the course of the reaction. Attempts to use stronger bases as $\mathrm{HCl}$ scavengers were not successful, as they proved incompatible with the acyliminium reagent. The structure of the products 5 was determined by NMR spectroscopy. The resolution of all NMR spectra of 5 at $25^{\circ} \mathrm{C}$ was very poor, which we assumed to be due to hindered rotation around the newly formed $C$ - $C$ bond. Indeed, when the NMR spectra were measured in DMSO at $80{ }^{\circ} \mathrm{C}$ their quality improved dramatically and assignment of peaks became possible. 


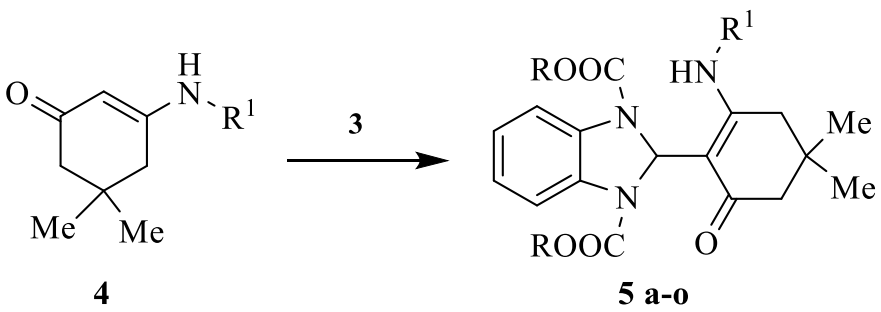

Scheme 2. Amidoalkylation of cyclic enaminones with benzimidazole-derived acyliminium reagents.

Table 1. Yields of compounds 5 obtained according to Scheme 2

\begin{tabular}{lllc}
\hline $\mathbf{5}$ & $\mathbf{R}$ & $\mathbf{R}^{\mathbf{1}}$ & Yield (\%) \\
\hline $\mathbf{a}$ & $\mathrm{C}_{2} \mathrm{H}_{5}$ & $3,4-\left(\mathrm{MeO}_{2} \mathrm{C}_{6} \mathrm{H}_{3} \mathrm{CH}_{2} \mathrm{CH}_{2}\right.$ & 55 \\
$\mathbf{b}$ & $\mathrm{C}_{2} \mathrm{H}_{5}$ & $\mathrm{C}_{6} \mathrm{H}_{5} \mathrm{CH}_{2} \mathrm{CH}_{2}$ & 40 \\
$\mathbf{c}$ & $\mathrm{C}_{2} \mathrm{H}_{5}$ & $\mathrm{C}_{6} \mathrm{H}_{5} \mathrm{CH}_{2}$ & 44 \\
$\mathbf{d}$ & $\mathrm{C}_{2} \mathrm{H}_{5}$ & $4-\mathrm{ClC}_{6} \mathrm{H}_{4} \mathrm{CH}_{2}$ & 44 \\
$\mathbf{e}$ & $\mathrm{C}_{2} \mathrm{H}_{5}$ & $\mathrm{C}_{6} \mathrm{H}_{5}$ & 28 \\
$\mathbf{f}$ & $\mathrm{C}_{2} \mathrm{H}_{5}$ & $\mathrm{C}_{3} \mathrm{H}_{7}$ & 32 \\
$\mathbf{g}$ & $\mathrm{C}_{2} \mathrm{H}_{5}$ & $\mathrm{C}_{2} \mathrm{H}_{5}$ & 17 \\
$\mathbf{h}$ & $\mathrm{C}_{2} \mathrm{H}_{5}$ & $\mathrm{CH}_{3}$ & 11 \\
$\mathbf{i}$ & $\mathrm{CH}_{3}$ & $3,4-\left(\mathrm{MeO}_{2} \mathrm{C}_{6} \mathrm{H}_{3} \mathrm{CH}_{2} \mathrm{CH}_{2}\right.$ & 21 \\
$\mathbf{j}$ & $\mathrm{CH}_{3}$ & $\mathrm{C}_{6} \mathrm{H}_{5} \mathrm{CH}_{2} \mathrm{CH}_{2}$ & 33 \\
$\mathbf{k}$ & $\mathrm{CH}_{3}$ & $\mathrm{C}_{6} \mathrm{H}_{5} \mathrm{CH}_{2}$ & 58 \\
$\mathbf{l}$ & $\mathrm{CH}_{3}$ & $4-\mathrm{ClC}_{6} \mathrm{H}_{4} \mathrm{CH}_{2}$ & 38 \\
$\mathbf{m}$ & $\mathrm{CH}_{3}$ & $\mathrm{C}_{6} \mathrm{H}_{5}$ & 35 \\
$\mathbf{n}$ & $\mathrm{CH}_{3}$ & $\mathrm{C}_{3} \mathrm{H}_{7}$ & 36 \\
$\mathbf{o}$ & $\mathrm{CH}_{3}$ & $\mathrm{C}_{2} \mathrm{H}_{5}$ & 41 \\
\hline
\end{tabular}

In conclusion, the scope of application of the intermolecular reaction of $\alpha$-amidoalkylation has been studied and series of cyclic enamino ketones were selectively amidoalkylated at the $\alpha$-carbon atom in reactions with acyliminium reagents derived from benzimidazole.

\section{Experimental Section}

General. Commercial solvents and reagents, such as benzimidazole and alkyl chloroformate were purchased from Sigma-Aldrich and were used without further purification. $\beta$-Enaminones were prepared according to a procedure described in the literature. ${ }^{19}$ Melting points were determined on a Boetius PHMKO5 hot stage apparatus and are uncorrected. ${ }^{1} \mathrm{H}-\mathrm{NMR}$ and ${ }^{13} \mathrm{C}-$ NMR spectra were measured on Bruker DRX250 and AV600 apparatus in DMSO as solvent. 
Chemical shifts are given in part per million (ppm) relative to TMS and coupling constants are indicated in Hz. All the NMR spectra were taken at $80^{\circ} \mathrm{C}$. TLC was done on precoated $0.2 \mathrm{~mm}$ Fluka silica gel 60 plates. Silica gel was used for column chromatographic separation.

General experimental procedure for preparation of compounds 5a-o: To a suspension of benzimidazole $(1 \mathrm{mmol})$ in 1,2-dichloroethane $(5 \mathrm{~mL})$ cooled to $0{ }^{\circ} \mathrm{C}$ were added $\mathrm{Et}_{3} \mathrm{~N}(1 \mathrm{mmol})$ and alkyl chloroformate $(1 \mathrm{mmol})$. The reaction mixture was stirred for $15 \mathrm{~min}$ at $0{ }^{\circ} \mathrm{C}$. To the same reaction mixture was added second equivalent of alkyl chloroformate $(1 \mathrm{mmol})$ and the mixture was stirred for $30 \mathrm{~min}$ at rt. Without isolation and purification of the formed $\mathrm{N}$ acyliminium reagent, to the reaction mixture was added equivalent amount of $\beta$-enaminone 4 . After $1 \mathrm{~h}$ water $(20 \mathrm{~mL})$ was added and the mixture was extracted with $3 \times 20 \mathrm{ml} \mathrm{CHCl}_{3}$. The combined organic layers were dried $\left(\mathrm{Na}_{2} \mathrm{SO}_{4}\right)$ and concentrated. After evaporation of the solvent the products were purified by column chromatography on silica gel using mixtures of petroleum and diethyl ether as eluents.

Diethyl 2-(2-((3,4-dimethoxyphenethyl)amino)-4,4-dimethyl-6-oxocyclohex-1-en-1-yl)-1Hbenzo[d]imidazole-1,3(2H)-dicarboxylate (5a). Mp 171-172 ${ }^{\circ} \mathrm{C}$; ${ }^{1} \mathrm{H}-\mathrm{NMR}: 0.95$ (s, 6H), 1.23 (t, 6H, J 7.1), $1.91(\mathrm{~s}, 2 \mathrm{H}), 2.31(\mathrm{~s}, 2 \mathrm{H}), 2.71(\mathrm{t}, 2 \mathrm{H}, J 6.9), 3.48(\mathrm{~m}, 2 \mathrm{H}), 3.75(\mathrm{~s}, 3 \mathrm{H}), 3.78$ (s, $3 \mathrm{H}), 4.14$ (q, 4H, J 7.1), 6.54 (br. s, 1H), 6.78 (d, 1H, J 8.0), 6.87-6.95 (m, 5H), 7.47-7.54 (m, 2H); ${ }^{13} \mathrm{C}-\mathrm{NMR}: 14.67,28.29,31.29,36.36,45.08,51.01,56.48,56.63,61.77,71.42,104.99$, 113.29, 113.74, 114.24, 121.48, 122.67, 131.97, 133.52, 148.62, 149.91, 151.43, 162.39, 191.01; Anal. Calcd. for $\mathrm{C}_{31} \mathrm{H}_{39} \mathrm{~N}_{3} \mathrm{O}_{7}$ : C, 65.82; H, 6.95; N, 7.43. Found: C, 65.60; H, 7.01; N, 7.38.

Diethyl 2-(4,4-dimethyl-6-oxo-2-(phenethylamino)cyclohex-1-en-1-yl)-1H-benzo[d]imidazole-1,3(2H)-dicarboxylate (5b). Mp 140-141 ${ }^{\circ} \mathrm{C}$; ${ }^{1} \mathrm{H}-\mathrm{NMR}: 0.95$ (s, 6H), 1.23 (t, 6H, J 7.0), 1.91 (s, 2H), $2.33(\mathrm{~s}, 2 \mathrm{H}), 2.79$ (t, 2H, J 7.5), $3.51(\mathrm{~m}, 2 \mathrm{H}), 4.14$ (q, 4H, J 7.0), 6.57 (br. s, 1H), 6.90-6.97 (m, 3H), 7.21-7.35 (m, 5H), 7.48-7.55 (m, 2H); ${ }^{13} \mathrm{C}-\mathrm{NMR}: 14.69,28.27,31.32$, 36.84, 44.89, 51.02, 61.76, 71.37, 105.06, 113.27, 122.67, 126.78, 128.86, 129.11, 133.54, 139.10, 151.41, 162.32, 191.05; Anal. Calcd. for $\mathrm{C}_{29} \mathrm{H}_{35} \mathrm{~N}_{3} \mathrm{O}_{5}$ : C, 68.89; H, 6.98; N, 8.31. Found: C, 68.72; H, 7.03; N, 8.28.

Diethyl 2-(2-(benzylamino)-4,4-dimethyl-6-oxocyclohex-1-en-1-yl)-1H-benzo[d]imidazole1,3(2H)-dicarboxylate (5c). Mp 165-166 ${ }^{\circ} \mathrm{C} ;{ }^{1} \mathrm{H}-\mathrm{NMR}: 0.39$ (s, 6H), 0.70 (t, 6H, J 7.0), 1.39 (s, 2H), 1.84 (s, 2H), 3.63 (q, 4H, J 7.0), 3.99 (d, 2H, J 4.2), 6.33-6.40 (m, 2H), 6.44 (s, 1H), 6.63 (br. s, 1H), 6.74 - $6.80(\mathrm{~m}, 1 \mathrm{H}), 6.84\left(\mathrm{~d}, 4 \mathrm{H}, J\right.$ 7.5), 6.93-7.00 (m, 2H); ${ }^{13} \mathrm{C}-\mathrm{NMR}: 14.75,28.24$, 31.41, 46.79, 51.05, 61.77, 71.37, 105.19, 113.24, 122.60, 127.39, 127.55, 128.86, 133.58, 139.42, 151.43, 162.49, 191.24; Anal. Calcd. for $\mathrm{C}_{28} \mathrm{H}_{33} \mathrm{~N}_{3} \mathrm{O}_{5}$ : C, 68.41; H, 6.77; N, 8.55. Found: C, 68.23; H, 6.81; N, 8.59.

Diethyl 2-(2-((4-chlorobenzyl)amino)-4,4-dimethyl-6-oxocyclohex-1-en-1-yl)-1H-benzo[d]imidazole-1,3(2H)-dicarboxylate (5d). Mp 168-169 ${ }^{\circ} \mathrm{C}$; ${ }^{1} \mathrm{H}$-NMR: 0.93 (s, 6H), 1.24 (t, 6H, J 7.0), 1.93 (s, 2H), 2.35 (s, 2H), 4.17 (q, 4H, J 7.0), 4.52 (d, 2H, J 5.3), 6.90-6.93 (m, 2H), 6.97 (s, 1H), 7.42 (s, 4H), 7.49 (br. s, 2H); ${ }^{13} \mathrm{C}-\mathrm{NMR}: 14.75,28.23,31.45,39.50,46.05$, 51.03, 61.80, 
71.33, 105.25, 113.23, 122.59, 128.82, 129.30, 132.35, 138.59, 151.41, 162.39, 191.35; Anal. Calcd. for $\mathrm{C}_{28} \mathrm{H}_{32} \mathrm{ClN}_{3} \mathrm{O}_{5}$ : C, 63.93; H, 6.13; N, 7.99. Found: C, 63.90; H, 6.21; N, 8.03.

Diethyl 2-(4,4-dimethyl-6-oxo-2-(phenylamino)cyclohex-1-en-1-yl)-1H-benzo[d]imidazole1,3(2H)-dicarboxylate (5e). Mp 184-187 ${ }^{\circ} \mathrm{C} ;{ }^{1} \mathrm{H}-\mathrm{NMR}: 0.96$ (s, 6H), 1.30 (t, 6H, J 7.1), 2.03 (s, 2H), 2.42 (s, 2H), 4.24 (q, 4H, J 7.1), 6.91-6.98 (m, 2H), $7.13(\mathrm{~s}, 1 \mathrm{H}), 7.17-7.22(\mathrm{~m}, 2 \mathrm{H}), 7.27$ (d, 1H, J 7.3), 7.42 - 7.48 (m, 2H), 7.50-7.55 (m, 2H), 8.84 (br. s, 1H); ${ }^{13} \mathrm{C}-\mathrm{NMR}: 14.35,27.48$, 31.84, 51.09, 61.43, 70.83, 107.54, 112.76, 122.10, 125.00, 125.18, 129.10, 133.31, 139.27, 150.98, 159.42, 192.24; Anal. Calcd. for $\mathrm{C}_{27} \mathrm{H}_{31} \mathrm{~N}_{3} \mathrm{O}_{5}$ : C, 67.91; H, 6.54; N, 8.80. Found: C, $67.95 ; \mathrm{H}, 6.60 ; \mathrm{N}, 7.78$.

Diethyl 2-(4,4-dimethyl-6-oxo-2-(propylamino)cyclohex-1-en-1-yl)-1H-benzo[ $d]$ imidazole1,3(2H)-dicarboxylate (5f): Mp 110-113 ${ }^{\circ} \mathrm{C} ;{ }^{1} \mathrm{H}-\mathrm{NMR}: 0.92(\mathrm{t}, 3 \mathrm{H}, J$ 7.4), $1.01(\mathrm{~s}, 6 \mathrm{H}), 1.24(\mathrm{t}$, 6H, J 7.1), 1.49 (sxt, 2H, J 7.1, 7.3), 1.95 (s, 2H), 2.39 (s, 2H), 3.18 - 3.26 (m, 2H), 4.16 (q, 4H, J 7.1), 6.42 (br. s, 1H), 6.91-6.98 (m, 3H), 7.50-7.57 (m, 2H); ${ }^{13} \mathrm{C}-\mathrm{NMR}: 10.91,14.16,23.15$, 27.82, 30.89, 44.68, 50.51, 61.21, 70.86, 104.33, 112.77, 122.18, 133.01, 150.92, 161.96, 190.36; Anal. Calcd. for $\mathrm{C}_{24} \mathrm{H}_{33} \mathrm{~N}_{3} \mathrm{O}_{5}$ : C, 64.99; H, 7.50; N, 9.47. Found: C, 65.07; H, 7.53; N, 9.41 .

Diethyl 2-(2-(ethylamino)-4,4-dimethyl-6-oxocyclohex-1-en-1-yl)-1H-benzo[d]imidazole1,3(2H)-dicarboxylate (5g). Mp 168-171 ${ }^{\circ} \mathrm{C}$; ${ }^{1} \mathrm{H}-\mathrm{NMR}: 1.01(\mathrm{~s}, 6 \mathrm{H}), 1.13(\mathrm{t}, 3 \mathrm{H}, J 7.1), 1.25(\mathrm{t}$, $6 \mathrm{H}, J 7.1), 1.94$ (s, 2H), 2.40 (s, 2H), 3.36-3.37 (m, 2H), 4.15 (q, 4H, J 7.1), 6.56 (br. s, 1H), 6.92-6.97 (m, 3H), 7.50-7.56 (m, 2H); ${ }^{13} \mathrm{C}-\mathrm{NMR}: 14.16,15.31,27.80,30.91,37.55,50.55$, 61.18, 70.80, 104.20, 112.64, 122.10, 133.15, 150.86, 161.92, 190.40; Anal. Calcd. for $\mathrm{C}_{23} \mathrm{H}_{31} \mathrm{~N}_{3} \mathrm{O}_{5}$ : C, 64.32; H, 7.27; N, 9.78. Found: C, 64.19; H, 7.30; N, 9.71.

Diethyl 2-(4,4-dimethyl-2-(methylamino)-6-oxocyclohex-1-en-1-yl)-1H-benzo[ $d]$ imidazole1,3(2H)-dicarboxylate (5h). Mp 198-200 ${ }^{\circ} \mathrm{C}$; ${ }^{1} \mathrm{H}-\mathrm{NMR}: 1.00$ (s, 6H), 1.24 (t, 6H, J 7.1), 1.91 (s, 2H), 2.39 (s, 2H), 2.95 (d, 3H, J 4.8), 4.14 (q, 4H, J 7.1), 6.80 (s, 1H), 6.89-6.94 (m, 2H), 7.10 (br. s, 1H), 7.46-7.54 (m, 2H); ${ }^{13} \mathrm{C}-\mathrm{NMR}: 14.09,27.87,29.81,30.82,50.62,61.16,70.87$, 104.20, 112.41, 121.85, 133.39, 150.79, 162.88, 190.38; Anal. Calcd. for $\mathrm{C}_{22} \mathrm{H}_{29} \mathrm{~N}_{3} \mathrm{O}_{5}$ : C, 63.60; H, 7.04; N, 10.11. Found: C, 63.57; H, 7.13; N, 10.05.

Dimethyl 2-(2-((3,4-dimethoxyphenethyl)amino)-4,4-dimethyl-6-oxocyclohex-1-en-1-yl)-1Hbenzo $[\boldsymbol{d}]$ imidazole-1,3(2H)-dicarboxylate (5i). Mp $168-173{ }^{\circ} \mathrm{C}$; ${ }^{1} \mathrm{H}-\mathrm{NMR}: 0.95(\mathrm{~s}, 6 \mathrm{H}), 1.91$ (s, 2H), 2.32 (s, 2H), $2.74(\mathrm{t}, 2 \mathrm{H}, J$ 7.1), 3.47 - $3.55(\mathrm{~m}, 2 \mathrm{H}), 3.70(\mathrm{~s}, 6 \mathrm{H}), 3.77(\mathrm{~s}, 3 \mathrm{H}), 3.80$ (s, 3H), 6.63 (br. s, $1 \mathrm{H}), 6.80$ (d, 1H, J 8.1), 6.89-6.98 (m, 5H), 7.46-7.51 (m, 2H); ${ }^{13} \mathrm{C}-\mathrm{NMR}$ : 27.67, 30.74, 35.98, 44.48, 50.48, 52.38, 55.98, 56.13, 71.05, 104.29, 112.77, 113.25, 113.79, 121.01, 122.23, 131.54, 132.91, 149.41, 151.36, 162.15, 190.58; Anal. Calcd. for $\mathrm{C}_{29} \mathrm{H}_{35} \mathrm{~N}_{3} \mathrm{O}_{7}$ : C, 64.79; H, 6.56; N, 7.82. Found: C, 64.75; H, 6.68; N, 7.80.

Dimethyl 2-(4,4-dimethyl-6-oxo-2-(phenethylamino)cyclohex-1-en-1-yl)-1H-benzo[d]imidazole-1,3(2H)-dicarboxylate (5j). Mp 139-141 ${ }^{\circ} \mathrm{C} ;{ }^{1} \mathrm{H}-\mathrm{NMR}: 0.95$ (s, 6H), 1.92 (s, 2H), 2.34 (s, 2H), 2.81 (t, 2H, J 7.3), $3.50-3.58$ (m, 2H), 3.70 (s, 6H), 6.66 (br. s, 1H), 6.90 (s, 1H), 6.93-6.98 (m, 2H), 7.25-7.37 (m, 5H), 7.47-7.54 (m, 2H); ${ }^{13} \mathrm{C}-\mathrm{NMR}: 28.16,31.26,36.93,44.79$, $50.98,52.88,71.50,104.86,113.27,122.73$, 126.75, 128.86, 129.13, 133.42, 139.17, 151.84, 
162.58, 191.12; Anal. Calcd. for $\mathrm{C}_{27} \mathrm{H}_{31} \mathrm{~N}_{3} \mathrm{O}_{5}$ : C, 67.91; H, 6.54; N, 8.80. Found: C, 67.72; $\mathrm{H}$, $6.61 ; \mathrm{N}, 8.69$.

Dimethyl 2-(2-(benzylamino)-4,4-dimethyl-6-oxocyclohex-1-en-1-yl)-1H-benzo[d]imidazole1,3(2H)-dicarboxylate (5k). Mp 180-184 ${ }^{\circ} \mathrm{C}$; ${ }^{1} \mathrm{H}-\mathrm{NMR}: 0.90$ (s, 6H), 1.92 (s, 2H), 2.37 (s, 2H), $3.70(\mathrm{~s}, 6 \mathrm{H}), 4.55$ (d, 2H, J 6.0), 6.88 - $6.94(\mathrm{~m}, 2 \mathrm{H}), 6.96(\mathrm{~s}, 1 \mathrm{H}), 7.26-7.34(\mathrm{~m}, 2 \mathrm{H}), 7.37-7.39$ (m, 4H), 7.45-7.51 (m, 2H); ${ }^{13} \mathrm{C}-\mathrm{NMR}: 28.13,31.37,46.68,51.02$, 52.88, 71.52, 104.93, 113.20, $122.64,127.25,127.52,128.92,133.54,139.62,151.86,162.79$, 191.33; Anal. Calcd. for $\mathrm{C}_{26} \mathrm{H}_{29} \mathrm{~N}_{3} \mathrm{O}_{5}$ : C, 67.37; H, 6.31; N, 9.07. Found: C, 67.31; H, 6.40; N, 9.12.

Dimethyl 2-(2-((4-chlorobenzyl)amino)-4,4-dimethyl-6-oxocyclohex-1-en-1-yl)-1H-benzo[d]imidazole-1,3(2H)-dicarboxylate (5l). Mp 172-175 ${ }^{\circ} \mathrm{C}$; ${ }^{1} \mathrm{H}-\mathrm{NMR}: 0.92$ (s, 6H), 1.94 (s, 2H), $2.36(\mathrm{~s}, 2 \mathrm{H}), 3.73(\mathrm{~s}, 6 \mathrm{H}), 4.56(\mathrm{~d}, 2 \mathrm{H}, J 6.1), 6.91-6.94(\mathrm{~m}, 2 \mathrm{H}), 6.97(\mathrm{~s}, 1 \mathrm{H}), 7.34(\mathrm{~s}, 1 \mathrm{H})$, 7.42-7.45 (m, 4H), 7.47-7.52 (m, 2H); ${ }^{13} \mathrm{C}-\mathrm{NMR}: 27.83,30.89,45.52,50.39$, 52.42, 70.95, 104.64, 112.71, 122.16, 128.39, 128.63, 131.83, 133.01, 138.18, 151.33, 162.37, 190.81; Anal. Calcd. for $\mathrm{C}_{26} \mathrm{H}_{28} \mathrm{ClN}_{3} \mathrm{O}_{5}$ : C, 62.71; H, 5.67; N, 8.44. Found: C, 62.59; H, 5.73; N, 8.39.

Dimethyl 2-(4,4-dimethyl-6-oxo-2-(phenylamino)cyclohex-1-en-1-yl)-1H-benzo[ $d$ ] imidazole1,3(2H)-dicarboxylate (5m). Mp 203-204 ${ }^{\circ} \mathrm{C} ;{ }^{1} \mathrm{H}-\mathrm{NMR}: 0.94(\mathrm{~s}, 6 \mathrm{H}), 2.01(\mathrm{~s}, 2 \mathrm{H}), 2.37$ (s, 2H), 3.78 (s, 6H), 6.91-6.98 (m, 2H), 7.09 (s, 1H), 7.17 - 7.29 (m, 3H), 7.42 - 7.45 (m, 2H), 7.49-7.52 (m, 2H), 8.86 (br. s, $1 \mathrm{H})$; ${ }^{13} \mathrm{C}-\mathrm{NMR}: 27.89,32.30,51.59$, 53.00, 71.50, 107.90, 113.18, 122.66, 125.92, 129.66, 133.70, 139.80, 151.85, 160.34, 192.74; Anal. Calcd. for $\mathrm{C}_{25} \mathrm{H}_{27} \mathrm{~N}_{3} \mathrm{O}_{5}$ : C, 66.80; H, 6.05; N, 9.35. Found: C, 66.73; H, 6.17; N, 9.31.

Dimethyl 2-(4,4-dimethyl-6-oxo-2-(propylamino)cyclohex-1-en-1-yl)-1H-benzo[d]imidazole1,3(2H)-dicarboxylate (5n). Mp 43-45 ${ }^{\circ} \mathrm{C} ;{ }^{1} \mathrm{H}-\mathrm{NMR}$ : 0.92 (t, 3H, J 7.2), 1.00 (s, 6H), 1.50 (sxt, $2 \mathrm{H}, J$ 7.1, 7.5), $1.95(\mathrm{~s}, 2 \mathrm{H}), 2.40(\mathrm{~s}, 2 \mathrm{H}), 3.20-3.28(\mathrm{~m}, 2 \mathrm{H}), 3.71(\mathrm{~s}, 6 \mathrm{H}), 6.52$ (br. s, 1H), 6.92-6.98 (m, 3H), 7.48-7.54 (m, 2H); ${ }^{13} \mathrm{C}-\mathrm{NMR}: 10.83,23.27,27.70,30.85,44.55,50.48$, 52.34, 71.00, 104.11, 112.77, 122.24, 132.89, 151.34, 162.24, 190.45; Anal. Calcd. for $\mathrm{C}_{22} \mathrm{H}_{29} \mathrm{~N}_{3} \mathrm{O}_{5}$ : C, 63.60; H, 7.04; N, 10.11. Found: C, 63.68; H, 7.13; N, 10.17 .

Dimethyl 2-(2-(ethylamino)-4,4-dimethyl-6-oxocyclohex-1-en-1-yl)-1H-benzo[d]imidazole1,3(2H)-dicarboxylate (5o). Mp 84-87 ${ }^{\circ} \mathrm{C}$; ${ }^{1} \mathrm{H}-\mathrm{NMR}: 1.00(\mathrm{~s}, 6 \mathrm{H}), 1.12$ (t, 3H, J 7.2), 1.94 (s, 2H), 2.40 (s, 2H), 3.27-3.37 (m, 2H), 3.71 (s, 6H), 6.60 (br. s, 1H), 6.92-6.98 (m, 3H), 7.477.54 (m, 2H); ${ }^{13} \mathrm{C}-\mathrm{NMR}: 15.48,27.70,30.85,37.48,50.52,52.32,70.98,104.11,112.68,122.18$, 132.99, 151.31, 162.13, 190.43; Anal. Calcd. for $\mathrm{C}_{21} \mathrm{H}_{27} \mathrm{~N}_{3} \mathrm{O}_{5}: \mathrm{C}, 62.83 ; \mathrm{H}, 6.78 ; \mathrm{N}, 10.47$. Found: C, 62.75; H, 6.83; N, 10.39 .

\section{Acknowledgements}

We acknowledge financial support from the fund for scientific research of Plovdiv University MU11 HF 003. 


\section{References}

1. Speckamp, W. N.; Moolenaar, M. J. Tetrahedron 2000, 56, 3817. http://dx.doi.org/10.1016/S0040-4020(00)00159-9

2. Hiemstra, H.; Speckamp, W. N. Comprehensive Organic Synthesis, Pergamon: Oxford, 1991, 2, p1047.

3. Maryanoff, B. E.; Zhang, H. C.; Cohen, J. H.; Turchi, I. J.; Maryanoff, C. A. Chem. Rev. 2004, 104, 1431.

http://dx.doi.org/10.1021/cr0306182

PMid:15008627

4. Yazici, A.; Pyne S. G. Synthesis 2009, 339.

5. Garuti, L.; Roberti, M.; Gentilomi, G. Il Farmaco 2000, 55, 35. http://dx.doi.org/10.1016/S0014-827X(99)00117-2

6. Goda, F. E.; Tantawy, A. S.; Abou-zeid, L.A.; Badr, S. M.; Selim, K. B. Saudi Pharmaceutical Journal 2008, 16 (2), 103.

7. Kamal, A.; Praveen Kumar, P.; Sreekanth, K.; Seshadri, B. N.; Ramulu, P. Bioorganic \& Medicinal Chemistry Letter, 2008, 18, 2594.

8. Abdel-Mohsen T. H.; Ragab F.; Ramla M. M.; El Diwani H. European Journal of Medicinal Chemistry 2010, 45, 2336. http://dx.doi.org/10.1016/j.ejmech.2010.02.011 PMid:20356655

9. Gowda, T.; Kavitha C. V.; Chiruvella K. K.; Joy O.; Rangappa S. K.; Raghavan C. S. Bioorganic \& Medicinal Chemistry Letters 2009, 19, 4594.

http://dx.doi.org/10.1016/j.bmcl.2009.06.103

PMid:19616939

10. Tulug, T. M. K.; Nurten, A. European Journal of Medicinal Chemistry 2009, 44, 1024. http://dx.doi.org/10.1016/j.ejmech.2008.06.026 PMid:18718694

11. Shingalapur, R. V.; Hosamani, K. M.; Keri, R. S. European Journal of Medicinal Chemistry 2009, 44, 4244.

http://dx.doi.org/10.1016/j.ejmech.2009.05.021

PMid:19540630

12. Jafar, A. A.; Vijayakumar, K. N.; Venkatramanb, B. R.; Venkatesha, G. Orbital 2009, 1 (4), 306.

13. Al-Tel, T. H.; Al-Qawasmeh, R. A. European Journal of Medicinal Chemistry 2010, 45, 5848.

http://dx.doi.org/10.1016/j.ejmech.2010.09.049

PMid:20934788 
14. Xue, F.; Luo, X.; Ye, C.; Ye, W.; Wang, Y. Bioorg. Med. Chem. 2011, 19, 2641. http://dx.doi.org/10.1016/j.bmc.2011.03.007 PMid:21441033

15. Statkova-Abeghe, S.; Angelov, P.; Ivanov, I.; Nikolova, S. Tetrahedron Letters 2007, 48, 6674.

http://dx.doi.org/10.1016/j.tetlet.2007.07.116

16. Venkov, A. P.; Statkova-Abeghe, S. M.; Donova, A. K. Cent. Eur. J. Chem. 2004, 2 (1), 234.

17. Donova, A. K.; Statkova-Abeghe, S. M.; Venkov, A. P.; Ivanov, I. Synth. Commun. 2004, 34 (15), 2813

18. Statkova-Abeghe S.; Ivanov, I.; Daskalova, S.; Dzhambazov, B. Med. Chem. Res. 2005, 429. http://dx.doi.org/10.1007/s00044-006-0147-0

19. Greenhill, J. V. Chem. Soc. Rev. 1977, 6, 277. http://dx.doi.org/10.1039/cs9770600277 